\title{
DETERMINANTAL FORMULAS AND RECURRENT RELATIONS FOR BI-PERIODIC FIBONACCI AND LUCAS POLYNOMIALS
}

\author{
FENG QI, EMRAH POLATLI, AND BAI-NI GUO \\ Dedicated to people facing and fighting COVID-19
}

\begin{abstract}
In the paper, the authors find closed formulas and recurrent relations for bi-periodic Fibonacci polynomials and for bi-periodic Lucas polynomials in terms of the Hessenberg determinants. Consequently, the authors derive closed formulas and recurrent relations for the Fibonacci, Lucas, bi-periodic Fibonacci, and bi-periodic Lucas numbers in terms of the Hessenberg determinants.
\end{abstract}

\section{Contents}

1. Introduction and preliminaries 1

2. Determinantal formulas 3

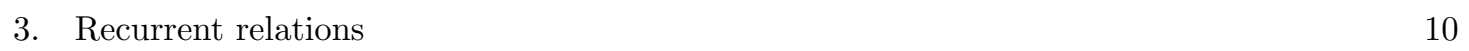

References 11

\section{INTRODUCTION AND PRELIMINARIES}

The Fibonacci and Lucas sequences are two important integer sequences which have been studied by many amateurs and professional mathematicians for centuries. There are numerous studies related to these sequences in the literature. They are defined respectively by recurrence relations

$$
F_{n+2}=F_{n+1}+F_{n} \quad \text { and } \quad L_{n+2}=L_{n+1}+L_{n}
$$

for $n \geq 0$, where $F_{0}=0, F_{1}=1, L_{0}=2$, and $L_{1}=1$. The values of the Fibonacci numbers $F_{n}$ and the Lucas numbers $L_{n}$ for $1 \leq n \leq 14$ are

$$
1, \quad 1, \quad 2, \quad 3, \quad 5, \quad 8, \quad 13, \quad 21, \quad 34, \quad 55, \quad 89, \quad 144, \quad 233, \quad 377
$$

and

$1, \quad 3, \quad 4, \quad 7, \quad 11, \quad 18, \quad 29, \quad 47, \quad 76, \quad 123, \quad 199, \quad 322, \quad 521, \quad 843$

respectively. See the monograph [6] and closely related references therein.

2010 Mathematics Subject Classification. Primary 11B39; Secondary 11B83, 11C20, 11Y55.

Key words and phrases. determinantal formula; recurrent relation; Hessenberg determinant; bi-periodic Fibonacci polynomial; bi-periodic Lucas polynomial; bi-periodic Fibonacci number; bi-periodic Lucas number; Fibonacci number; Lucas number.

This paper was typeset using $\mathcal{A} \mathcal{M} \mathcal{S}$ - $\mathrm{LAT}_{\mathrm{E}} \mathrm{X}$. 
There are many generalizations for the Fibonacci sequence in the literature. An interesting one of them was given in [4] by

$$
q_{0}=0, \quad q_{1}=1, \quad q_{n}= \begin{cases}a q_{n-1}+q_{n-2}, & \text { if } n \text { is even } \\ b q_{n-1}+q_{n-2}, & \text { if } n \text { is odd }\end{cases}
$$

for $n \geq 2$, where $a$ and $b$ are two nonzero real numbers. Various identities and other results for the sequence $\left\{q_{n}\right\}_{n=0}^{\infty}$ were given in [4]. What we are interested in this paper is the generating function

$$
\frac{x\left(1+a x-x^{2}\right)}{1-(a b+2) x^{2}+x^{4}}=\sum_{n=0}^{\infty} q_{n} x^{n}=x+a x^{2}+(a b+1) x^{3}+a(a b+2) x^{4}+\cdots
$$

derived in 4 .

In [1, the Lucas sequence was generalized by

$$
\ell_{0}=2, \quad \ell_{1}=a, \quad \ell_{n}= \begin{cases}a \ell_{n-1}+\ell_{n-2}, & \text { if } n \text { is odd } \\ b \ell_{n-1}+\ell_{n-2}, & \text { if } n \text { is even }\end{cases}
$$

for $n \geq 2$, where $a$ and $b$ are two nonzero real numbers. One of main results in [1] is the generating function

$$
\frac{2+a x-(a b+2) x^{2}+a x^{3}}{1-(a b+2) x^{2}+x^{4}}=\sum_{n=0}^{\infty} \ell_{n} x^{n}=2+a x+(a b+2) x^{2}+a(a b+3) x^{3}+\cdots
$$

In [25, bi-periodic Fibonacci and Lucas polynomials are defined respectively as

$$
q_{0}(x)=0, \quad q_{1}(x)=1, \quad q_{n}(x)= \begin{cases}a x q_{n-1}(x)+q_{n-2}(x), & \text { if } n \text { is even } \\ b x q_{n-1}(x)+q_{n-2}(x), & \text { if } n \text { is odd }\end{cases}
$$

and

$$
\ell_{0}(x)=2, \quad \ell_{1}(x)=a x, \quad \ell_{n}(x)= \begin{cases}b x \ell_{n-1}(x)+\ell_{n-2}(x), & \text { if } n \text { is even } \\ a x \ell_{n-1}(x)+\ell_{n-2}(x), & \text { if } n \text { is odd }\end{cases}
$$

for $n \geq 2$, where $a$ and $b$ are any two nonzero real numbers. The generating functions

$$
\begin{gathered}
Q(t ; x)=\frac{t+a x t^{2}-t^{3}}{1-\left(2+a b x^{2}\right) t^{2}+t^{4}}=\sum_{n=0}^{\infty} q_{n}(x) t^{n} \\
=t-a x t^{2}+\left(a b x^{2}+1\right) t^{3}-a x\left(a b x^{2}+2\right) t^{4}+\left(a^{2} b^{2} x^{4}+3 a b x^{2}+1\right) t^{5}+\cdots
\end{gathered}
$$

and

$$
\begin{gathered}
L(t ; x)=\frac{2+a x t-\left(2+a b x^{2}\right) t^{2}+a x t^{3}}{1-\left(2+a b x^{2}\right) t^{2}+t^{4}}=\sum_{n=0}^{\infty} \ell_{n}(x) t^{n} \\
=2+a x t+\left(a b x^{2}+2\right) t^{2}+a x\left(a b x^{2}+3\right) t^{3}+\left(a^{2} b^{2} x^{4}+4 a b x^{2}+2\right) t^{4}+\cdots
\end{gathered}
$$

were also discovered in [25].

Inheriting from terminologies for $q_{n}(x)$ and $\ell_{n}(x)$, we call $q_{n}$ and $\ell_{n}$ defined in 1.2 and 1.3 bi-periodic Fibonacci numbers and bi-periodic Lucas numbers respectively. 
A determinant $|A|=\left|a_{i j}\right|_{n \times n}$ is called a lower (or an upper, respectively) Hessenberg determinant if and only if $a_{i j}=0$ for all pairs $(i, j)$ such that $i+1<j$ (or $j+1<i$, respectively). For more details, please refer to [5, 7] and closely related references therein.

In the paper, we will find closed formulas and recurrent relations for bi-periodic Fibonacci polynomials $q_{n}(x)$ and for bi-periodic Lucas polynomials $\ell_{n}(x)$ in terms of some Hessenberg determinants. Consequently, we will derive closed formulas and recurrent relations for bi-periodic Fibonacci numbers $q_{n}$, for bi-periodic Lucas numbers $\ell_{n}$, for the Fibonacci numbers $F_{n}$, and for the Lucas numbers $L_{n}$ in terms of some Hessenberg determinants.

\section{Determinantal formulas}

Our main results can be stated and proved as follows.

Theorem 2.1. For $n \geq 0$, bi-periodic Fibonacci polynomials $q_{n}(x)$ can be formulated determinantally as

$$
\begin{aligned}
& q_{n}(x)=\frac{(-1)^{n}}{n !} \mid \begin{array}{ccccc}
0 & 1 & 0 & 0 & \cdots \\
1 & 0 & 1 & 0 & \cdots \\
2 a x & -2\left(\begin{array}{l}
2 \\
0
\end{array}\right)\left(2+a b x^{2}\right) & 0 & 1 & \cdots \\
-6 & 0 & -2\left(\begin{array}{c}
3 \\
1
\end{array}\right)\left(2+a b x^{2}\right) & 0 & \cdots \\
0 & 24\left(\begin{array}{l}
4 \\
0
\end{array}\right) & 0 & -2\left(\begin{array}{l}
4 \\
2
\end{array}\right)\left(2+a b x^{2}\right) & \cdots \\
0 & 0 & 24\left(\begin{array}{l}
5 \\
1
\end{array}\right) & 0 & \cdots \\
0 & 0 & 0 & 24\left(\begin{array}{c}
6 \\
2
\end{array}\right) & \cdots \\
\vdots & \vdots & \vdots & \vdots & \ddots \\
0 & 0 & 0 & 0 & \cdots \\
0 & 0 & 0 & 0 & \cdots \\
0 & 0 & 0 & 0 & \cdots
\end{array} \\
& \begin{array}{cccc}
0 & 0 & 0 & 0 \\
0 & 0 & 0 & 0 \\
0 & 0 & 0 & 0 \\
0 & 0 & 0 & 0 \\
0 & 0 & 0 & 0 \\
0 & 0 & 0 & 0 \\
0 & 0 & 0 & 0 \\
\vdots & \vdots & \vdots & \vdots \\
-2\left(\begin{array}{c}
n-2 \\
n-4
\end{array}\right)\left(2+a b x^{2}\right) & 0 & 1 & 0 \\
0 & -2\left(\begin{array}{c}
n-1 \\
n-3
\end{array}\right)\left(2+a b x^{2}\right) & 0 & 1 \\
24\left(\begin{array}{c}
n \\
n-4
\end{array}\right) & 0 & -2\left(\begin{array}{c}
n \\
n-2
\end{array}\right)\left(2+a b x^{2}\right) & 0
\end{array}
\end{aligned}
$$

Proof. In [10, Section 2.2, p. 849], [13, p. 94], and [24, Lemma 2.1], Exercise 5) in [2, p. 40] was reformulated as the following conclusion. Let $u(t)$ and $v(t) \neq 0$ be two differentiable functions. Let $U_{(n+1) \times 1}(t)$ be an $(n+1) \times 1$ matrix whose elements $u_{k, 1}(t)=u^{(k-1)}(t)$ for $1 \leq k \leq n+1$, let $V_{(n+1) \times n}(t)$ be an $(n+1) \times n$ matrix whose elements $v_{i, j}(t)=\left(\begin{array}{c}i-1 \\ j-1\end{array}\right) v^{(i-j)}(t)$ for $1 \leq i \leq n+1$ 
and $1 \leq j \leq n$, and let $\left|W_{(n+1) \times(n+1)}(t)\right|$ denote the determinant of the $(n+1) \times(n+1)$ matrix $W_{(n+1) \times(n+1)}(t)=\left(U_{(n+1) \times 1}(t) \quad V_{(n+1) \times n}(t)\right)$. Then the $n$th derivative of the ratio $\frac{u(t)}{v(t)}$ can be computed by

$$
\frac{\mathrm{d}^{n}}{\mathrm{~d} x^{n}}\left[\frac{u(t)}{v(t)}\right]=(-1)^{n} \frac{\left|W_{(n+1) \times(n+1)}(t)\right|}{v^{n+1}(t)} .
$$

Letting $u(t)=t+a x t^{2}-t^{3}$ and $v(t)=1-\left(2+a b x^{2}\right) t^{2}+t^{4}$ in 2.1 yields

$$
\begin{aligned}
& t+a x t^{2}-t^{3} \quad 1-\left(2+a b x^{2}\right) t^{2}+t^{4} \\
& 1+2 a x t-3 t^{2} \quad\left(\begin{array}{l}
1 \\
0
\end{array}\right)\left[4 t^{3}-2\left(2+a b x^{2}\right) t\right] \\
& 2 a x-6 t \quad\left(\begin{array}{l}
2 \\
0
\end{array}\right)\left[12 t^{2}-2\left(2+a b x^{2}\right)\right] \\
& -6 \quad 24\left(\begin{array}{l}
3 \\
0
\end{array}\right) t \\
& \frac{\partial^{n} Q(t ; x)}{\partial t^{n}}=\frac{(-1)^{n}}{\left[1-\left(2+a b x^{2}\right) t^{2}+t^{4}\right]^{n+1}} \mid \begin{array}{cc}
0 & 24\left(\begin{array}{c}
4 \\
0
\end{array}\right) \\
0 & 0 \\
0 & \vdots \\
\vdots & 0 \\
0 & 0 \\
0 & 0 \\
0 &
\end{array} \\
& \begin{array}{cccc}
0 & 0 & \cdots & 0
\end{array} \\
& 1-\left(2+a b x^{2}\right) t^{2}+t^{4} \quad 0 \quad 0 \quad \ldots
\end{aligned}
$$

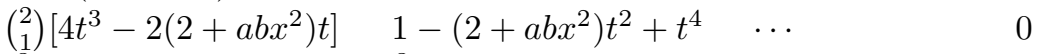

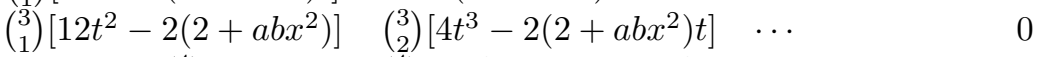

$$
\begin{aligned}
& \begin{array}{llll}
24\left(\begin{array}{l}
4 \\
1
\end{array}\right) t & \left(\begin{array}{l}
4 \\
2
\end{array}\right)\left[12 t^{2}-2\left(2+a b x^{2}\right)\right] & \cdots & 0
\end{array}
\end{aligned}
$$

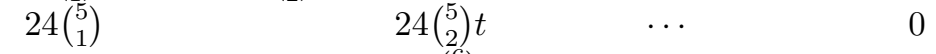

$$
\begin{aligned}
& \begin{array}{llll}
0 & 24\left(\begin{array}{c}
6 \\
2
\end{array}\right) & \cdots & 0
\end{array} \\
& \begin{array}{llll}
\vdots & \vdots & \ddots & \vdots
\end{array} \\
& \begin{array}{llll}
0 & 0 & \cdots & \left(\begin{array}{c}
n-2 \\
n-4
\end{array}\right)\left[12 t^{2}-2\left(2+a b x^{2}\right)\right]
\end{array} \\
& \begin{array}{llll}
0 & 0 & \cdots & 24\left(\begin{array}{c}
n-1 \\
n-4
\end{array}\right) t
\end{array} \\
& \begin{array}{llll}
0 & 0 & \cdots & 24\left(\begin{array}{c}
n \\
n-4
\end{array}\right)
\end{array} \\
& \begin{array}{ccc|}
0 & 0 & 0 \\
0 & 0 & 0 \\
0 & 0 & 0 \\
0 & 0 & 0 \\
0 & 0 & 0 \\
0 & 0 & 0 \\
0 & 0 & 0 \\
\vdots & \vdots & \vdots \\
\left(\begin{array}{c}
n-2 \\
n-3
\end{array}\right)\left[4 t^{3}-2\left(2+a b x^{2}\right) t\right] & 1-\left(2+a b x^{2}\right) t^{2}+t^{4} & 0 \\
\left(\begin{array}{c}
n-1 \\
n-3
\end{array}\right)\left[12 t^{2}-2\left(2+a b x^{2}\right)\right] & \left(\begin{array}{c}
n-1 \\
n-2
\end{array}\right)\left[4 t^{3}-2\left(2+a b x^{2}\right) t\right] & 1-\left(2+a b x^{2}\right) t^{2}+t^{4} \\
24\left(\begin{array}{c}
n \\
n-3
\end{array}\right) t & \left(\begin{array}{c}
n \\
n-2
\end{array}\right)\left[12 t^{2}-2\left(2+a b x^{2}\right)\right] & \left(\begin{array}{c}
n \\
n-1
\end{array}\right)\left[t^{3}-2\left(2+a b x^{2}\right) t\right]
\end{array}
\end{aligned}
$$


Letting $t \rightarrow 0$ for $n \in \mathbb{N}$ in the above equation, we obtain

$$
\begin{aligned}
& \lim _{t \rightarrow 0} \frac{\partial^{n} Q(t ; x)}{\partial t^{n}}=(-1)^{n} \mid \begin{array}{cccc}
0 & 1 & 0 & 0 \\
1 & 0 & 1 & 0 \\
2 a x & -2\left(\begin{array}{l}
2 \\
0
\end{array}\right)\left(2+a b x^{2}\right) & 0 & 1 \\
-6 & 0 & -2\left(\begin{array}{l}
3 \\
1
\end{array}\right)\left(2+a b x^{2}\right) & 0 \\
0 & 24\left(\begin{array}{l}
4 \\
0
\end{array}\right) & 0 & -2\left(\begin{array}{l}
4 \\
2
\end{array}\right)\left(2+a b x^{2}\right) \\
0 & 0 & 24\left(\begin{array}{l}
5 \\
1
\end{array}\right) & 0 \\
0 & 0 & 0 & 24\left(\begin{array}{l}
6 \\
2
\end{array}\right) \\
\vdots & \vdots & \vdots & \vdots \\
0 & 0 & 0 & 0 \\
0 & 0 & 0 & 0 \\
0 & 0 & 0 & 0
\end{array} \\
& \begin{array}{lcccc}
\ldots & 0 & 0 & 0 & 0 \\
\ldots & 0 & 0 & 0 & 0 \\
\ldots & 0 & 0 & 0 & 0 \\
\ldots & 0 & 0 & 0 & 0 \\
\ldots & 0 & 0 & 0 & 0 \\
\ldots & 0 & 0 & 0 & 0 \\
\ldots & 0 & 0 & 0 & 0 \\
\ddots & \vdots & \vdots & \vdots & \vdots \\
\ldots & -2\left(\begin{array}{c}
n-2 \\
n-4
\end{array}\right)\left(2+a b x^{2}\right) & 0 & 1 & 0 \\
\cdots & 0 & -2\left(\begin{array}{c}
n-1 \\
n-3
\end{array}\right)\left(2+a b x^{2}\right) & 0 & 1 \\
\cdots & 24\left(\begin{array}{c}
n \\
n-4
\end{array}\right) & 0 & -2\left(\begin{array}{c}
n \\
n-2
\end{array}\right)\left(2+a b x^{2}\right) & 0
\end{array}
\end{aligned}
$$

The proof of Theorem 2.1 is thus complete.

Corollary 2.1. The bi-periodic Fibonacci numbers $q_{n}$ and the Fibonacci numbers $F_{n}$ for $n \geq 0$ can be determinantally formulated by

$$
q_{n}=\frac{(-1)^{n}}{n !} \mid \begin{array}{ccccc}
0 & 1 & 0 & 0 & \cdots \\
1 & 0 & 1 & 0 & \cdots \\
2 a & -2\left(\begin{array}{c}
2 \\
0
\end{array}\right)(2+a b) & 0 & 1 & \cdots \\
-6 & 0 & -2\left(\begin{array}{c}
3 \\
1
\end{array}\right)(2+a b) & 0 & \cdots \\
0 & 24\left(\begin{array}{l}
4 \\
0
\end{array}\right) & 0 & -2\left(\begin{array}{c}
4 \\
2
\end{array}\right)(2+a b) & \cdots \\
0 & 0 & 24\left(\begin{array}{c}
5 \\
1
\end{array}\right) & 0 & \cdots \\
0 & 0 & 0 & 24\left(\begin{array}{c}
6 \\
2
\end{array}\right) & \cdots \\
\vdots & \vdots & \vdots & \vdots & \ddots \\
0 & 0 & 0 & 0 & \cdots \\
0 & 0 & 0 & 0 & \cdots \\
0 & 0 & 0 & 0 & \cdots
\end{array}
$$




$\begin{array}{cccc}0 & 0 & 0 & 0 \\ 0 & 0 & 0 & 0 \\ 0 & 0 & 0 & 0 \\ 0 & 0 & 0 & 0 \\ 0 & 0 & 0 & 0 \\ 0 & 0 & 0 & 0 \\ 0 & 0 & 0 & 0 \\ \vdots & \vdots & \vdots & \vdots \\ -2\left(\begin{array}{c}n-2 \\ n-4\end{array}\right)(2+a b) & 0 & 1 & 0 \\ 0 & -2\left(\begin{array}{c}n-1 \\ n-3\end{array}\right)(2+a b) & 0 & 1 \\ 24\left(\begin{array}{c}n \\ n-4\end{array}\right) & 0 & -2\left(\begin{array}{c}n \\ n-2\end{array}\right)(2+a b) & 0\end{array} \mid$.

and

$$
F_{n}=\frac{(-1)^{n}}{n !}\left|\begin{array}{ccccccccc}
0 & 1 & 0 & 0 & \cdots & 0 & 0 & 0 & 0 \\
1 & 0 & 1 & 0 & \cdots & 0 & 0 & 0 & 0 \\
2 & -6\left(\begin{array}{c}
2 \\
0
\end{array}\right) & 0 & 1 & \cdots & 0 & 0 & 0 & 0 \\
-6 & 0 & -6\left(\begin{array}{c}
3 \\
1
\end{array}\right) & 0 & \cdots & 0 & 0 & 0 & 0 \\
0 & 24\left(\begin{array}{c}
4 \\
0
\end{array}\right) & 0 & -6\left(\begin{array}{l}
4 \\
2
\end{array}\right) & \cdots & 0 & 0 & 0 & 0 \\
0 & 0 & 24\left(\begin{array}{c}
5 \\
1
\end{array}\right) & 0 & \cdots & 0 & 0 & 0 & 0 \\
0 & 0 & 0 & 24\left(\begin{array}{c}
6 \\
2
\end{array}\right) & \cdots & 0 & 0 & 0 & 0 \\
\vdots & \vdots & \vdots & \vdots & \ddots & \vdots & \vdots & \vdots & \vdots \\
0 & 0 & 0 & 0 & \cdots & -6\left(\begin{array}{c}
n-2 \\
n-4
\end{array}\right) & 0 & 1 & 0 \\
0 & 0 & 0 & 0 & \cdots & 0 & -6\left(\begin{array}{c}
n-1 \\
n-3
\end{array}\right) & 0 & 1 \\
0 & 0 & 0 & 0 & \cdots & 24\left(\begin{array}{c}
n \\
n-4
\end{array}\right) & 0 & -6\left(\begin{array}{c}
n \\
n-2
\end{array}\right) & 0
\end{array}\right| .
$$

Proof. These follow from taking $x \rightarrow 1$ and letting $(a, b) \rightarrow(1,1)$ in Theorem 2.1 respectively.

Remark 2.1. The determinantal formula (2.2) is different from those collected in [9, 12, 16, 21] and closely related references therein.

Theorem 2.2. For $n \in\{0\} \cup \mathbb{N}$, bi-periodic Lucas polynomials $\ell_{n}(x)$ can be formulated determinantally as

$$
\ell_{n}(x)=\frac{(-1)^{n}}{n !} \mid \begin{array}{ccccc}
2 & 1 & 0 & 0 & \cdots \\
a x & 0 & 1 & 0 & \cdots \\
-2\left(2+a b x^{2}\right) & -2\left(\begin{array}{c}
2 \\
0
\end{array}\right)\left(2+a b x^{2}\right) & 0 & 1 & \cdots \\
6 a x & 0 & -2\left(\begin{array}{c}
3 \\
1
\end{array}\right)\left(2+a b x^{2}\right) & 0 & \cdots \\
0 & 24\left(\begin{array}{l}
4 \\
0
\end{array}\right) & 0 & -2\left(\begin{array}{l}
4 \\
2
\end{array}\right)\left(2+a b x^{2}\right) & \cdots \\
0 & 0 & 24\left(\begin{array}{c}
5 \\
1
\end{array}\right) & 0 & \cdots \\
0 & 0 & 0 & 24\left(\begin{array}{c}
6 \\
2
\end{array}\right) & \cdots \\
\vdots & \vdots & \vdots & \vdots & \ddots \\
0 & 0 & 0 & 0 & \cdots \\
0 & 0 & 0 & 0 & \cdots \\
0 & 0 & 0 & 0 & \cdots
\end{array}
$$




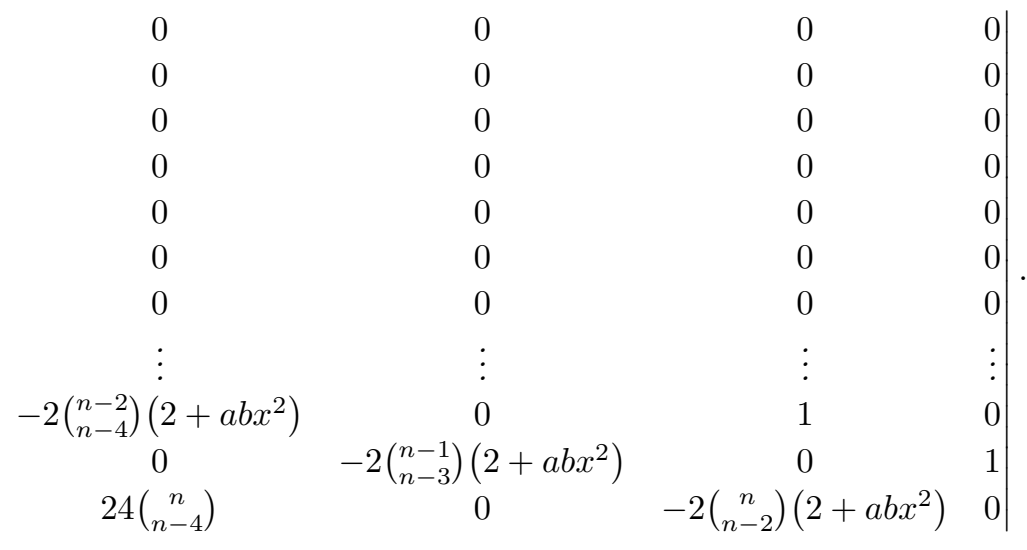

Proof. Applying $u(t)=2+a x t-\left(2+a b x^{2}\right) t^{2}+a x t^{3}$ and $v(t)=1-\left(2+a b x^{2}\right) t^{2}+t^{4}$ in 2.1) results in

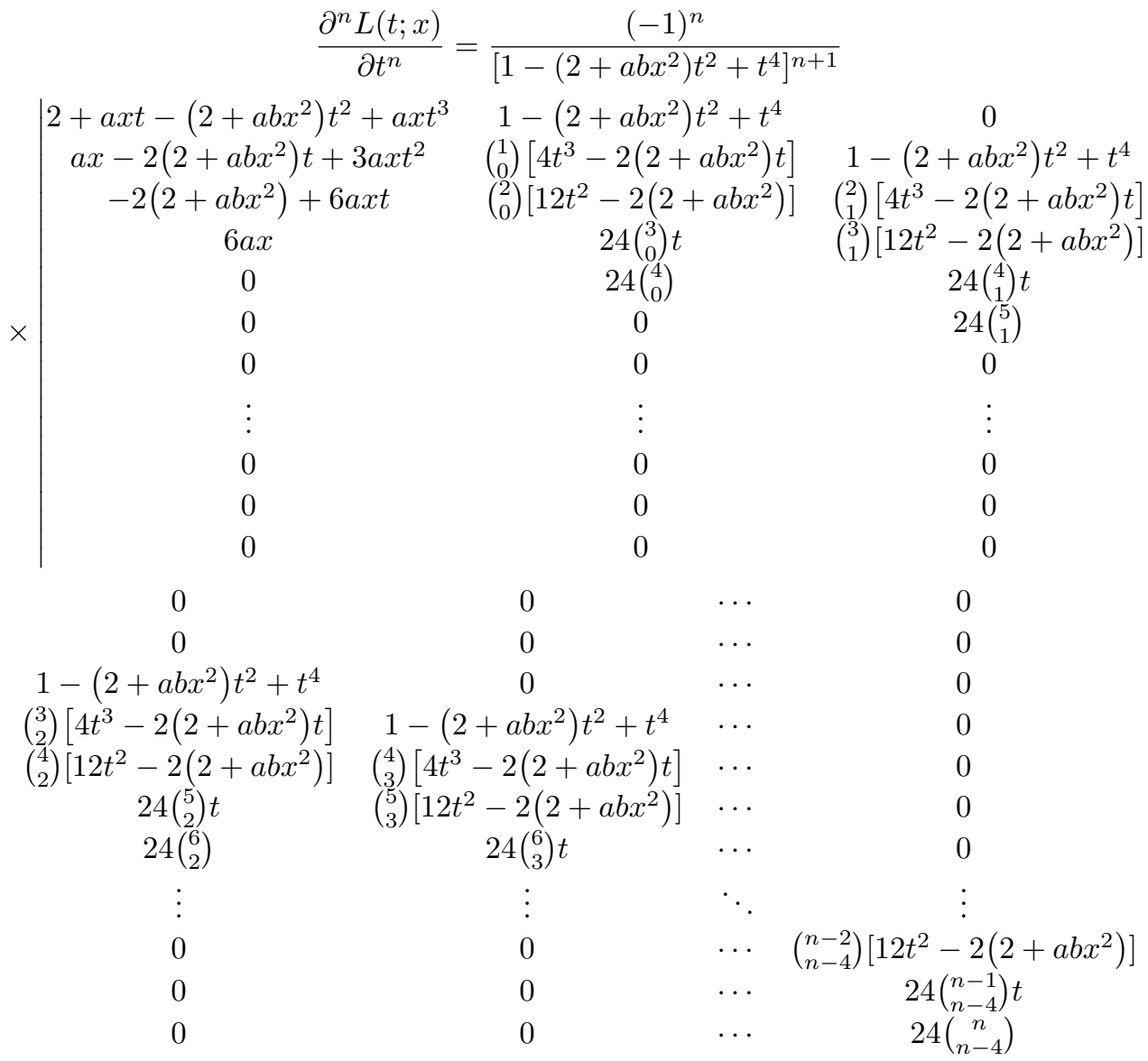




\begin{tabular}{|c|c|c|}
\hline 0 & 0 & 0 \\
\hline 0 & 0 & 0 \\
\hline 0 & 0 & 0 \\
\hline 0 & 0 & 0 \\
\hline 0 & 0 & 0 \\
\hline 0 & 0 & 0 \\
\hline 0 & 0 & 0 \\
\hline$\vdots$ & $\vdots$ & $\vdots$ \\
\hline $\begin{array}{c}\left(\begin{array}{l}n-2 \\
n-3\end{array}\right)\left[4 t^{3}-2\left(2+a b x^{2}\right) t\right] \\
\left(\begin{array}{l}n-1 \\
n-3\end{array}\right)\left[12 t^{2}-2\left(2+a b x^{2}\right)\right] \\
24\left(\begin{array}{c}n \\
n-3\end{array}\right) t\end{array}$ & $\begin{array}{c}1-\left(2+a b x^{2}\right) t^{2}+t^{4} \\
\left(\begin{array}{c}n-1 \\
n-2\end{array}\right)\left[4 t^{3}-2\left(2+a b x^{2}\right) t\right] \\
\left(\begin{array}{c}n \\
n-2\end{array}\right)\left[12 t^{2}-2\left(2+a b x^{2}\right)\right]\end{array}$ & $\begin{array}{c}0 \\
1-\left(2+a b x^{2}\right) t^{2}+t^{4} \\
\left(\begin{array}{c}n \\
n-1\end{array}\right)\left[4 t^{3}-2\left(2+a b x^{2}\right) t\right]\end{array}$ \\
\hline
\end{tabular}

Letting $t \rightarrow 0$ in the above equation yields

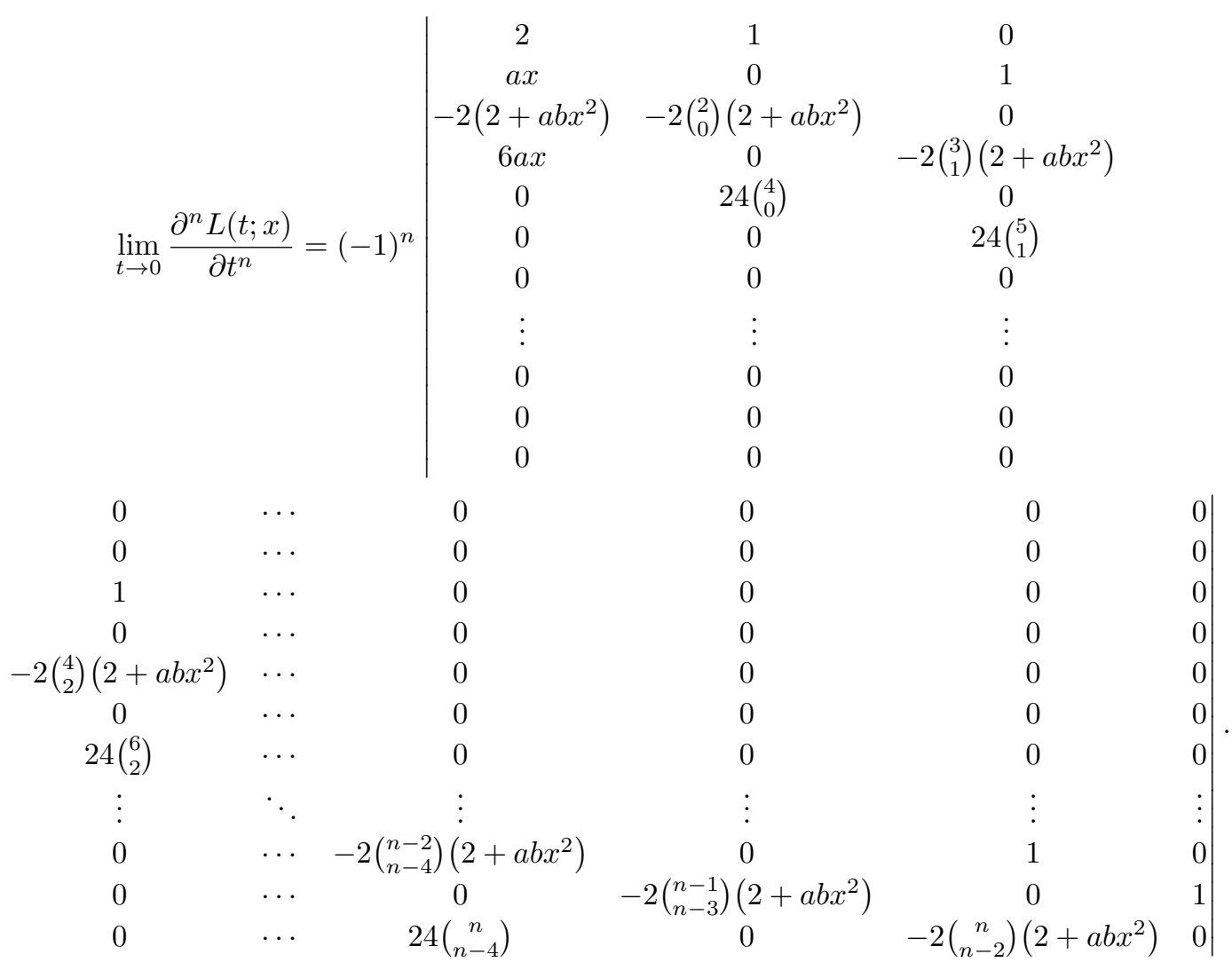

The proof of Theorem 2.2 is thus complete. 
Corollary 2.2. The bi-periodic Lucas numbers $\ell_{n}$ and the Lucas numbers $L_{n}$ for $n \geq 0$ can be determinantally formulated by

$$
\ell_{n}=\frac{(-1)^{n}}{n !} \mid \begin{array}{ccccc}
2 & 1 & 0 & 0 & \cdots \\
a & 0 & 1 & 0 & \cdots \\
-2(2+a b) & -2\left(\begin{array}{c}
2 \\
0
\end{array}\right)(2+a b) & 0 & 1 & \cdots \\
6 a & 0 & -2\left(\begin{array}{c}
3 \\
1
\end{array}\right)(2+a b) & 0 & \cdots \\
0 & 24\left(\begin{array}{c}
4 \\
0
\end{array}\right) & 0 & -2\left(\begin{array}{c}
4 \\
2
\end{array}\right)(2+a b) & \cdots \\
0 & 0 & 24\left(\begin{array}{l}
5 \\
1
\end{array}\right) & 0 & \cdots \\
0 & 0 & 0 & 24\left(\begin{array}{c}
6 \\
2
\end{array}\right) & \cdots \\
\vdots & \vdots & \vdots & \vdots & \ddots \\
0 & 0 & 0 & 0 & \cdots \\
0 & 0 & 0 & 0 & \cdots \\
0 & 0 & 0 & 0 & \cdots
\end{array}
$$

$\begin{array}{cccc}0 & 0 & 0 & 0 \\ 0 & 0 & 0 & 0 \\ 0 & 0 & 0 & 0 \\ 0 & 0 & 0 & 0 \\ 0 & 0 & 0 & 0 \\ 0 & 0 & 0 & 0 \\ 0 & 0 & \vdots & 0 \\ \vdots & \vdots & 1 & \vdots \\ -2\left(\begin{array}{c}n-2 \\ n-4\end{array}\right)(2+a b) & 0 & 0 & 1 \\ 0 & -2\left(\begin{array}{c}n-1 \\ n-3\end{array}\right)(2+a b) & 0 & 0 \\ 24\left(\begin{array}{c}n \\ n-4\end{array}\right) & 0 & & 0\end{array}$

and

$$
L_{n}=\frac{(-1)^{n}}{n !}\left|\begin{array}{ccccccccc}
2 & 1 & 0 & 0 & \cdots & 0 & 0 & 0 & 0 \\
1 & 0 & 1 & 0 & \cdots & 0 & 0 & 0 & 0 \\
-6 & -6\left(\begin{array}{c}
2 \\
0
\end{array}\right) & 0 & 1 & \cdots & 0 & 0 & 0 & 0 \\
6 & 0 & -6\left(\begin{array}{l}
3 \\
1
\end{array}\right) & 0 & \cdots & 0 & 0 & 0 & 0 \\
0 & 24\left(\begin{array}{c}
4 \\
0
\end{array}\right) & 0 & -6\left(\begin{array}{c}
4 \\
2
\end{array}\right) & \cdots & 0 & 0 & 0 & 0 \\
0 & 0 & 24\left(\begin{array}{c}
5 \\
1
\end{array}\right) & 0 & \cdots & 0 & 0 & 0 & 0 \\
0 & 0 & 0 & 24\left(\begin{array}{c}
6 \\
2
\end{array}\right) & \cdots & 0 & 0 & 0 & 0 \\
\vdots & \vdots & \vdots & \vdots & \ddots & \vdots & \vdots & \vdots & \vdots \\
0 & 0 & 0 & 0 & \cdots & -6\left(\begin{array}{c}
n-2 \\
n-4
\end{array}\right) & 0 & 1 & 0 \\
0 & 0 & 0 & 0 & \cdots & 0 & -6\left(\begin{array}{c}
n-1 \\
n-3
\end{array}\right) & 0 & 1 \\
0 & 0 & 0 & 0 & \cdots & 24\left(\begin{array}{c}
n \\
n-4
\end{array}\right) & 0 & -6\left(\begin{array}{c}
n \\
n-2
\end{array}\right) & 0
\end{array}\right| .
$$

Proof. These follow from taking $x \rightarrow 1$ and letting $(a, b) \rightarrow(1,1)$ in Theorem 2.2 respectively.

Remark 2.2. For more information on applications of the formula (2.1), please refer to the papers 8 , 11, 13, 14, 17, 18, 19, 23, 24] and closely related references therein. 


\section{RECURRENT RELATiOns}

In this section, we derive, by a different method, several recurrent relations.

Theorem 3.1. For $n \geq 5$, we have the recurrent relations

$$
\begin{gathered}
q_{n+4}(x)=\left(2+a b x^{2}\right) q_{n+2}(x)-q_{n}(x), \\
\ell_{n+4}(x)=\left(2+a b x^{2}\right) \ell_{n+2}(x)-\ell_{n}(x), \\
q_{n+4}=(2+a b) q_{n+2}-q_{n}, \quad \ell_{n+4}=(2+a b) \ell_{n+2}-\ell_{n},
\end{gathered}
$$

and

$$
F_{n+4}=3 F_{n+2}-F_{n}, \quad L_{n+4}=3 L_{n+2}-L_{n}
$$

Proof. Let $D_{0}=1$ and

$$
D_{n}=\left|\begin{array}{cccccc}
e_{1,1} & e_{1,2} & 0 & \ldots & 0 & 0 \\
e_{2,1} & e_{2,2} & e_{2,3} & \ldots & 0 & 0 \\
e_{3,1} & e_{3,2} & e_{3,3} & \ldots & 0 & 0 \\
\vdots & \vdots & \vdots & \vdots & \vdots & \vdots \\
e_{n-2,1} & e_{n-2,2} & e_{n-2,3} & \ldots & e_{n-2, n-1} & 0 \\
e_{n-1,1} & e_{n-1,2} & e_{n-1,3} & \ldots & e_{n-1, n-1} & e_{n-1, n} \\
e_{n, 1} & e_{n, 2} & e_{n, 3} & \ldots & e_{n, n-1} & e_{n, n}
\end{array}\right|
$$

for $n \in \mathbb{N}$. In [3, p. 222, Theorem], it was proved that the sequence $D_{n}$ for $n \geq 0$ satisfies $D_{1}=e_{1,1}$ and

$$
D_{n}=\sum_{r=1}^{n}(-1)^{n-r} e_{n, r}\left(\prod_{j=r}^{n-1} e_{j, j+1}\right) D_{r-1}
$$

for $n \geq 2$, where the empty product is understood to be 1. See also [15, Lemma 5], 17, Lemma 2], [22, Lemma 2], and [23, Remark 3]. Applying the recurrent relation (3.5) to Theorem 2.1 reveals that

$$
\begin{aligned}
&(-1)^{n-1}(n-1) ! q_{n-1}(x)=2\left(\begin{array}{l}
n-1 \\
n-3
\end{array}\right)\left(2+a b x^{2}\right)(-1)^{n-3}(n-3) ! q_{n-3}(x) \\
&-24\left(\begin{array}{c}
n-1 \\
n-5
\end{array}\right)(-1)^{n-5}(n-5) ! q_{n-5}(x)
\end{aligned}
$$

for $n \geq 10$, which can be simplified as 3.1 . .

Similarly, applying the recurrent relation 3.5 to Theorem 2.2 and simplifying lead to the recurrent relation 3.2 .

The recurrent relations in (3.3) and (3.4) follows from taking $x \rightarrow 1$ and letting $(a, b) \rightarrow(1,1)$ in (3.1) and 3.2 respectively. The proof of Theorem 3.1 is complete.

Remark 3.1. The recurrent relations 3.1), (3.2), 3.3), and (3.4) in Theorem 3.1 are different from corresponding ones in 1.1), (1.2), 1.3), 1.4, and (1.5).

Remark 3.2. Some of recurrent relations in Theorem 3.1 appeared in [1, Lemma 1], 4, p. 643], and [25, Eq. (7)]. 
Remark 3.3. This paper is a slightly revised version of the electronic preprint [20].

\section{REFERENCES}

[1] G. Bilgici, Two generalizations of Lucas sequence, Appl. Math. Comput. 245 (2014), 526-538; available online at https://doi.org/10.1016/j.amc.2014.07.111

[2] N. Bourbaki, Elements of Mathematics: Functions of a Real Variable: Elementary Theory, Translated from the 1976 French original by Philip Spain. Elements of Mathematics (Berlin). Springer-Verlag, Berlin, 2004; available online at http://dx.doi.org/10.1007/978-3-642-59315-4

[3] N. D. Cahill, J. R. D'Errico, D. A. Narayan, and J. Y. Narayan, Fibonacci determinants, College Math. J. 33 (2002), no. 3, 221-225; available online at http://dx.doi.org/10.2307/1559033

[4] M. Edson and O. Yayenie, A new generalization of Fibonacci sequence and extended Binet's formula, Integers 9 (2009), A48, 639-654; available online at https://doi.org/10.1515/INTEG.2009.051

[5] V. Hingins and C. Johnson, Inverse spectral problems for collections of leading principal submatrices of tridiagonal matrices, Linear Algebra Appl. 489 (2016), 104-122; available online at https://doi.org/10.1016/j. 1aa.2015.10.004

[6] T. Koshy, Fibonacci and Lucas Numbers with Applications, Pure and Applied Mathematics (New York). WileyInterscience, New York, 2001; available online at https://doi.org/10.1002/9781118033067

[7] R. S. Martin and J. H. Wilkinson, Handbook Series Linear Algebra: Similarity reduction of a general matrix to Hessenberg form, Numer. Math. 12 (1968), no. 5, 349-368; available online at https://doi.org/10.1007/ BF02161358.

[8] F. Qi, A determinantal expression and a recursive relation of the Delannoy numbers, arXiv preprint (2020), available online at https://arxiv.org/abs/2003.12572v1

[9] F. Qi, Denying a short proof of a determinantal formula for generalized Fibonacci polynomials, J. Math. Anal. 11 (2020), no. 1, 52-57.

[10] F. Qi, Derivatives of tangent function and tangent numbers, Appl. Math. Comput. 268 (2015), 844-858; available online at http://dx.doi.org/10.1016/j.amc.2015.06.123

[11] F. Qi, Determinantal expressions and recurrence relations for Fubini and Eulerian polynomials, J. Interdiscip. Math. 22 (2019), no. 3, 317-335; available online at https://doi.org/10.1080/09720502.2019.1624063

[12] F. Qi, V. Čerňanová, and Y. S. Semenov, Some tridiagonal determinants related to central Delannoy numbers, the Chebyshev polynomials, and the Fibonacci polynomials, Politehn. Univ. Bucharest Sci. Bull. Ser. A Appl. Math. Phys. 81 (2019), no. 1, 123-136.

[13] F. Qi and R. J. Chapman, Two closed forms for the Bernoulli polynomials, J. Number Theory 159 (2016), 89-100; available online at https://doi.org/10.1016/j.jnt.2015.07.021

[14] F. Qi and B.-N. Guo, A determinantal expression and a recurrence relation for the Euler polynomials, Adv. Appl. Math. Sci. 16 (2017), no. 9, 297-309.

[15] F. Qi and B.-N. Guo, A diagonal recurrence relation for the Stirling numbers of the first kind, Appl. Anal. Discrete Math. 12 (2018), no. 1, 153-165; available online at https://doi.org/10.2298/AADM170405004Q

[16] F. Qi and B.-N. Guo, Expressing the generalized Fibonacci polynomials in terms of a tridiagonal determinant, Matematiche (Catania) 72 (2017), no. 1, 167-175; available online at https://doi.org/10.4418/2017.72.1.13

[17] F. Qi and B.-N. Guo, Some determinantal expressions and recurrence relations of the Bernoulli polynomials, Mathematics 4 (2016), no. 4, Article 65, 11 pages; available online at https://doi.org/10.3390/math4040065.

[18] F. Qi and B.-N. Guo, Two nice determinantal expressions and a recurrence relation for the Apostol-Bernoulli polynomials, J. Indones. Math. Soc. (MIHMI) 23 (2017), no. 1, 81-87; available online at https://doi.org/10. 22342/jims.23.1.274.81-87

[19] F. Qi, C. Kızılateş, and W.-S. Du, A closed formula for the Horadam polynomials in terms of a tridiagonal determinant, Symmetry 11 (2019), no. 6, 8 pages; available online at https://doi.org/10.3390/sym11060782

[20] F. Qi, E. Polatli, and B.-N. Guo, Determinantal formulas and recurrent relations for bi-periodic Fibonacci and Lucas polynomials, OSF Preprints (2020), available online at https://doi.org/10.31219/osf.io/qvxd8 
[21] F. Qi, J.-L. Wang, and B.-N. Guo, A determinantal expression for the Fibonacci polynomials in terms of a tridiagonal determinant, Bull. Iranian Math. Soc. 45 (2019), no. 6, 1821-1829; available online at https: //doi.org/10.1007/s41980-019-00232-4

[22] F. Qi and J.-L. Zhao, Some properties of the Bernoulli numbers of the second kind and their generating function, Bull. Korean Math. Soc. 55 (2018), no. 6, 1909-1920; available online at https://doi.org/10.4134/BKMS . b180039.

[23] F. Qi, J.-L. Zhao, and B.-N. Guo, Closed forms for derangement numbers in terms of the Hessenberg determinants, Rev. R. Acad. Cienc. Exactas Fís. Nat. Ser. A Mat. RACSAM 112 (2018), no. 4, 933-944; available online at https://doi.org/10.1007/s13398-017-0401-z

[24] C.-F. Wei and F. Qi, Several closed expressions for the Euler numbers, J. Inequal. Appl. 2015, Paper No. 219, 8 pages; available online at https://doi.org/10.1186/s13660-015-0738-9

[25] N. Yılmaz, A. Coşkun, and N. Taşkara, On properties of bi-periodic Fibonacci and Lucas polynomials, AIP Conference Proceedings 1863, 310002 (2017); available online at https://doi.org/10.1063/1.4992478

(Qi) College of Mathematics, Inner Mongolia University for Nationalities, Tongliao 028043, Inner Mongolia, China; Institute of Mathematics, Henan Polytechnic University, Jiaozuo 454010, Henan, China; School of Mathematical Sciences, Tianjin Polytechnic University, Tianjin 300387, China

Email address: qifeng618@gmail.com, qifeng618@hotmail.com, qifeng618@qq.com

$U R L:$ https://qifeng618. wordpress.com

(Polatli) Department Of Mathematics, Zonguldak Bülent Ecevit University, Zonguldak 67100, Turkey Email address: emrahpolatli@gmail.com $U R L:$ https://orcid.org/0000-0002-2349-8978

(Guo) School of Mathematics and Informatics, Henan Polytechnic University, Jiaozuo 454010, Henan, CHINA

Email address: bai.ni.guo@gmail.com, bai.ni.guo@hotmail.com

$U R L$ : https://orcid.org/0000-0001-6156-2590 